\title{
An Improved Car-Following Model considering Desired Safety Distance and Heterogeneity of Driver's Sensitivity
}

\author{
Lei Zhang $\mathbb{D})^{1,2}$ Shengrui Zhang $\mathbb{D}^{1},{ }^{1}$ Bei Zhou $\mathbb{D}^{\mathbb{D}},{ }^{1}$ Shuaiyang Jiao $\mathbb{D}^{1},{ }^{1}$ and Yan Huang ${ }^{1}$ \\ ${ }^{1}$ College of Transportation Engineering, Chang'an University, Xi'an 710064, China \\ ${ }^{2}$ School of Civil and Transportation Engineering, Henan University of Urban Construction, Pingdingshan 467036, China
}

Correspondence should be addressed to Shengrui Zhang; zhangsr@chd.edu.cn

Received 20 October 2020; Revised 29 December 2020; Accepted 18 January 2021; Published 29 January 2021

Academic Editor: Ludovic Leclercq

Copyright (c) 2021 Lei Zhang et al. This is an open access article distributed under the Creative Commons Attribution License, which permits unrestricted use, distribution, and reproduction in any medium, provided the original work is properly cited.

We investigate the dynamic performance of traffic flow using a modified optimal velocity car-following model. In the carfollowing scenarios, the following vehicle must continuously adjust the following distance to the preceding vehicle in real time. A new optimal velocity function incorporating the desired safety distance instead of a preset constant is presented first to describe the abovementioned car-following behavior dynamically. The boundary conditions of the new optimal velocity function are theoretically analyzed. Subsequently, we propose an improved car-following model by combining the heterogeneity of driver's sensitivity based on the new optimal velocity function and previous car-following model. The stability criterion of the improved model is obtained through the linear analysis method. Finally, numerical simulation is performed to explore the effect of the desired safety distance and the heterogeneity of driver's sensitivity on the traffic flow. Results show that the proposed model has considerable effects on improving traffic stability and suppressing traffic congestion. Furthermore, the proposed model is compatible with the heterogeneity of driver's sensitivity and can enhance the average velocity of traffic flow compared with the conventional model. In conclusion, the dynamic performance of traffic flow can be improved by considering the desired safety distance and the heterogeneity of driver's sensitivity in the car-following model.

\section{Introduction}

In recent years, many cities worldwide have experienced traffic congestion and the accompanying traffic safety issues, which have attracted considerable attention from numerous scholars. Traffic models, such as car-following, cellular automation, gas kinetic, and hydrodynamic lattice models, have been extensively studied to understand the complicated constitution behind the traffic congestion in traffic flow [1-4].

Car-following model is a favorable microscopic traffic model to describe vehicles' longitudinal interactions on the road in studying traffic flow. In the early 1950s, Reuschel [5] and Pipes [6] first developed the stimulus-response model, which assumed that the driver attempted to adjust the following vehicle's velocity for consistency with that of the preceding vehicle. Kometani and Sasaki [7] proposed a safety distance car-following model for a driver to maintain a reasonable safety distance for avoiding collisions when they cannot fully predict the movement of the preceding vehicle. Kikuchi and Chakroborty [8] used fuzzy logic inference theory to establish an artificial intelligence car-following model. Newell [9] introduced the velocity function into the car-following model by assuming a corresponding relationship between the velocity and the space headway. Later, Bando et al. [10] proposed a popular optimal velocity carfollowing model (OVM), which has successfully described the stop-and-go phenomenon, traffic instability, and traffic congestion. In 2000, one popular model using desired measures was the intelligent driver model proposed by Treiber et al. [11]. More recently, arising high-fidelity traffic data provide an opportunity to model car-following behavior directly from a large number of field data by using machine learning techniques [12]. Among these models, the OVM is generally used to explore the traffic flow properties because it is straightforward and descriptive. 
Many scholars are devoted to investigating vehicles' longitudinal interactions as inspired by the OVM for alleviating traffic congestion and improving traffic flow stability. Helbing et al. [13] presented a generalized force model (GFM) by considering the negative velocity difference based on OVM. However, Treiber et al. [11] argued that the OVM and GFM could not explain why the following vehicle will not brake if the preceding vehicle is much faster even if its space headway is smaller than the safe distance. Jiang et al. [14] put forward a full velocity difference model (FVDM) based on the GFM by considering the negative and positive differences. Subsequently, many extended car-following models have been presented by incorporating different factors, such as the preceding vehicle's velocity $[15,16]$, multiple preceding vehicles' velocities [17], the velocity difference [18], the acceleration difference [19], and the historical velocity difference [20].

Vehicle distance is a direct stimulus that can typically trigger the driver's response during car-following. Nagatani et al. [21] believed that the following vehicle's behavior is affected by the space headway with the preceding vehicle and the vehicle interaction before the next vehicle ahead. Lenz et al. [22] established an optimal velocity function by calculating the average space headway of multiple preceding vehicles and found that the proposed model can improve the traffic flow stability. Ge et al. [23] developed a similarly car-following model by assigning different weights to multiple preceding vehicles' space headway. The analysis results showed that the traffic flow became stable. On this basis, Nakayama [24] explored the backward-looking optimal velocity model with consideration of the immediately following vehicle's space headway. Later, Hasebe [25] investigated the stability of uniform flow and the response to a disturbance in the linear approximation using the information of an arbitrary number of vehicles that precede or follow. In addition to space headway, the safety distance is an important indicator. Notably, Xin et al. [26] explored the performance of traffic flow by considering the variable time headway policies to modify the safety distance in the optimal velocity function. They identified that variable gap policies with proper parameters directly contribute to improving the traffic flow's stability. Cheng et al. [27] put forward that the optimal velocity function's safety distance should be a variable. Thus, they modified it into a random statistic to extend the model. The safety distance also plays an essential role in the car-following model. However, less research has focused on this issue.

More recently, the driver's characteristics have been found to have a significant influence on car-following behavior. Liu et al. [28] demonstrated that considering the driver's short-term memory's predictive action could improve traffic flow stability in the car-following behavior. Furthermore, Zhai et al. [29] presented an extended continuum model by considering the optimal velocity changes with memory. The analysis results showed that traffic efficiency was improved and energy consumption was minimized. Zhai et al. [30] incorporated the driver's selfanticipation characteristics into the OVM and analyzed the effect of the driver's predictive behavior on traffic flow. Other modified models have introduced drivers' characteristics, such as aggressiveness, cautious features [31], and time delay [32]. These studies have attempted to explore their advances in describing human driving behavior during car-following. However, different drivers have different driving styles and reactions [33, 34]. Ossen et al. [35] used a large sample of trajectory observations to explore the driving behaviors' heterogeneity. Schultz et al. [36] identified that log-normal and normal distribution alternatives were very effective in representing the full distribution of driver's sensitivity. Makridis et al. [37] showed that the heterogeneity of the vehicle-driver system could affect traffic dynamics and reproduce traffic oscillations. These existing studies can provide a useful reference and help for subsequent research. However, research on modeling the car-following behavior based on the heterogeneity of driver's sensitivity is limited to our knowledge.

According to the research mentioned above, the dynamic safety distance and the heterogeneity of driver's sensitivity have rarely been considered in previous carfollowing models. The optimal velocity function's safety distance is always assumed to be constant and independent of velocity. In reality, the safety distance changes dynamically depending on the real-time velocity of the vehicle, which is based on the driver's estimation of the realistic traffic situation. The driver must adjust the safety distance to the preceding vehicle in real time to avoid a potential collision. This process involves two critical features: one is that the safety distance varies in real time, and the other is that the individual driver's sensitivity has unobserved heterogeneity. In most studies, the driver's sensitivity is assumed to be a constant for convenience. This assumption is not practical for the driver's characteristics. In reality, it cannot be identical for all drivers. This study aims to explore the effect of desired safety distance and the heterogeneity of driver's sensitivity on the car-following behavior to reveal a more realistic and reasonable traffic flow performance. Therefore, an improved car-following model considering the desired safety distance and the heterogeneity of driver's sensitivity is proposed based on OVM. The dynamic performance of the proposed car-following model on the stability of traffic flow is explored.

The rest of the paper is organized as follows. In Section 2, a new optimal velocity function is first given. Then, an improved car-following model that combines the heterogeneity of driver's sensitivity is proposed based on the OVM. The linear analysis of the stability condition of the improved model is presented in Section 3. The numerical simulations are conducted to explore the performance of the improved model in Section 4. Conclusions are provided in Section 5.

\section{Modeling}

2.1. Previous Car-Following Models. In 1961, Newell [9] assumed that the vehicle's velocity at time $t$ is some function of the space headway at time $t-\Delta t$, and a particular velocityspace headway relation is shown in equation (1).

$$
v_{n}(t)=G_{n}\left(x_{n-1}(t-\Delta t)-x_{n}(t-\Delta t)\right),
$$


where $v_{n}(t)$ is the velocity of the $n$th vehicle at time $t$, $x_{n-1}(t-\Delta t)-x_{n}(t-\Delta t)$ is the space headway, and $G_{n}$ is an exponential function. The model establishes a function of velocity and space headway. However, the acceleration tends toward infinity when the vehicle starts accelerating.

In 1995, Bando et al. [10] proposed an optimal velocity model to overcome Newell's excessive acceleration problem. The authors concentrated on the dynamic evolution of congestion, which is formulated as follows:

$$
a_{n}=\kappa\left[V\left(\Delta x_{n}(t)\right)-v_{n}\right],
$$

where $a_{n}$ and $v_{n}$ are the acceleration and velocity of vehicle $n$, respectively; $\Delta x_{n}(t)$ is the space headway of vehicle $n ; \kappa$ is a constant representing the driver's sensitivity. The optimal velocity $V\left(\Delta x_{n}(t)\right)$ is a function that depends on the following distance of the preceding vehicle. $V\left(\Delta x_{n}(t)\right)$ can be expressed as equation (3).

$$
V\left(\Delta x_{n}(t)\right)=\frac{v_{\max }}{2}\left[\tanh \left((\Delta) x_{n}(t)-h_{c}\right)+\tanh h_{c}\right],
$$

where $v_{\max }$ is the maximum velocity and $h_{c}$ is the safety distance, which is a constant in the model. However, the safety distance should be updated dynamically in real carfollowing scenarios. The drivers need to adjust the following vehicle's velocity according to the desired safety distance, which is estimated by their driving experience and the preceding vehicle's motion. A significant variation could be triggered by the heterogeneity of the diver's sensitivity. This performance cannot be well displayed in equations (2) and (3). Therefore, exploring an improved car-following model is necessary.

2.2. New Optimal Velocity Function. In general, drivers want to maintain the desired safety distance $h_{v}$ in real time to avoid accelerating and decelerating frequently. A driver's decision to accelerate or decelerate is determined by the difference between the space headway and the desired safety distance, which can be expressed as $\Delta x_{n}(t)-h_{v}$ in coincidence with a part of equation (3). When $\Delta x_{n}(t)>h_{v}$, the following vehicle accelerates and keeps up with the vehicle in front. When $\Delta x_{n}(t)<h_{v}$, the following vehicle decelerates to avoid a collision. When $\Delta x_{n}(t)=h_{v}$, the following vehicle maintains a constant velocity. Thus, the desired safety distance's accuracy directly determines the acceleration output, which is the core of the car-following model.

Calculating the desired safety distance is crucial. Velocity is the most direct and essential factor that affects the safety distance between vehicles. Instead of a preset constant, the desired safety distance should depend on the vehicles' velocity in real time during car-following. The following vehicle's driver should keep the desired safety distance for avoiding traffic collisions during the preceding vehicle emergency braking [38]. The desired safety distance can be shown in Figure 1. (4):

The desired safety distance can be calculated as equation

$$
h_{v}=x_{n-1}-x_{n}=d_{1}+d_{2}+h_{0}-d_{3} .
$$

Equation (4) can be further expressed as follows:

$$
h_{v}=v_{n} t_{0}+\frac{v_{n}^{2}}{2 a_{\max }}-\frac{v_{n-1}^{2}}{2 a_{\max }}+h_{0},
$$

where $x_{n-1}$ and $x_{n}$ denote the positions of the preceding and following vehicles, respectively; $h_{v}$ denotes the desired safety distance; $d_{1}$ denotes the distance of vehicle $n$ within the response time $t_{0}$, which is generally in the order of 1s [39]. This threshold is higher than the 99\% quantile of the expected response time during the car-following; thus, it can meet the driver's safety requirements; $d_{2}$ denotes the braking distance of vehicle $n ; d_{3}$ denotes the braking distance of vehicle $n-1 ; h_{0}$ denotes the parking safety distance; $v_{n-1}$ and $v_{n}$ are the velocities of the preceding and following vehicles, respectively; and $a_{\max }$ is the maximum braking deceleration. As shown in Figure 1, it is more realistic to car-following behavior as the desired safety distance updates depending on the vehicle's velocity. Therefore, we attempt to replace the constant $h_{c}$ in the optimal velocity function equation (3) with $h_{v}$ in equation (5). In this way, the new optimal velocity function depends on the space headway, and the desired safety distance can be expressed as follows:

$$
V\left(\Delta x_{n}(t), h_{v}\right)=\frac{v_{\max }}{2}\left[\tanh \left(\Delta x_{n}(t)-h_{v}\right)+\tanh \left(h_{v}\right)\right] .
$$

Then, the property of the optimal velocity equation (6) should be discussed. The partial derivative is obtained concerning $\Delta x$, which can be obtained as follows:

$$
\frac{\partial V\left(\Delta x_{n}(t), h_{v}\right)}{\partial \Delta x_{n}(t)}=\frac{v_{\max }}{2}\left(1-\tanh ^{2}\left(\Delta x_{n}(t)-h_{v}\right)\right) .
$$

Similarly, the partial derivative with respect to $h_{v}$ is obtained as follows:

$$
\frac{\partial V\left(\Delta x_{n}(t), h_{v}\right)}{\partial h_{v}}=\frac{v_{\max }}{2}\left(2+\tanh ^{2}\left(\Delta x_{n}(t)-h_{v}\right)-\tanh ^{2}\left(h_{v}\right)\right) .
$$

From equations (7) and (8), we can obtain equations (9) and (10):

$$
\begin{aligned}
& \frac{\partial \mathrm{V}\left(\Delta x_{n}(\mathrm{t}), h_{v}\right)}{\partial \Delta x_{n}(\mathrm{t})} \geq 0, \\
& \frac{\partial \mathrm{V}\left(\Delta x_{n}(\mathrm{t}), h_{v}\right)}{\partial h_{v}} \geq 0 .
\end{aligned}
$$

From equations (9) and (10), the following properties can be inferred:

(i) $V\left(\Delta x_{n}(t), h_{v}\right)$ is a monotonically increasing function.

(ii) The change rate of the optimal velocity is related to the space headway and the desired safety distance.

(iii) When $\Delta x_{n}(t) \longrightarrow \infty$, the optimal velocity $V(\infty)=\left(v_{\max } / 2\right)\left[1+\tanh \left(h_{v}\right)\right]$. When $h_{v} \gg 0$, the optimal velocity tends to be maximized, indicating 


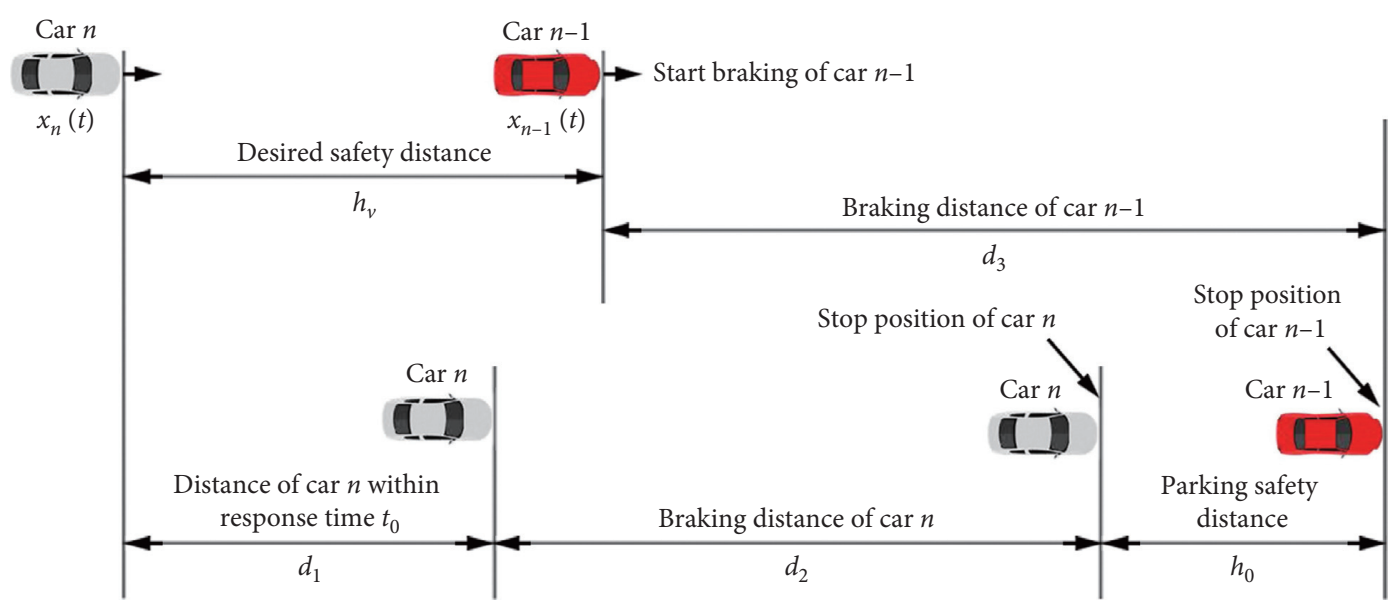

Figure 1: Schematic of the desired safety distance of the car-following scenarios during emergency braking.

that the optimal velocity function has an upper bound.

2.3. Heterogeneity of Driver's Sensitivity. Heterogeneity of driver's sensitivity plays an essential role during car-following due to its enormous influence on traffic flow. Differences between the longitudinal driving behaviors of drivers determine the flow-dependent distribution of vehicles over lanes to a large extent [35]. Although several traffic simulation models have considered multiple driver classes to accurately describe heterogeneous perception and preferences, the most widely used car-following models still assume the driver's sensitivity as constant. Particularly, study results regarding these parameter values are inconsistent. Some are listed in Table 1, such as those of Bando et al. [10], Helbing et al. [13], Jiang et al. [14], and Ge et al. [40].

Although the heterogeneity of driver's sensitivity has a significant effect on traffic instability and congestion, it is not straightforward for modeling because of its stochastic nature. The sensitivity varies with the extent of external stimulation. It should be a random parameter that depends on the driver's characteristics within a specific range. A particular distribution might describe the parameter. Song et al. [20] pointed out that the driver's sensitivity corresponds to the inverse of the response time. This relationship is also consistent with the classical differential equation of the linear car-following model [6]. Therefore, we quantify the driver's sensitivity with the inverse of the response time. The driver's sensitivity equals the inverse of the response time; that is, $\kappa=1 / \tau$, where $\tau$ is the response time distribution.

The response time is a critical controlling factor of the driving performance of the vehicle. The value of the response time is also individually varying and bounded [41]. The information on response time is available in real traffic. Research has shown that response times obey a log-normal distribution [42]. According to the relationship that the driver's sensitivity corresponds to the inverse of the response time, we conclude that the driver's sensitivity is also subject to the log-normal distribution to account for its individualspecific heterogeneity. This finding is consistent with the results of Schultz et al. [36], which consider generating an input parameter from a log-normal distribution of central tendency and dispersion in traffic simulation models. The distribution of sensitivity parameters may better reflect real traffic behavior than previous studies and have a practical physical meaning. According to empirical analysis, the response time obeys the log-normal distribution of equation (11). $\eta$ and $\xi$ are the two parameters that define the shape of the distribution. The statistical experience values of $\mu$ and $\sigma$ are 1.31 and 0.61 , respectively [42].

$$
\begin{aligned}
f(\tau) & =\frac{1}{\sqrt{2 \pi} \xi \tau} \exp \left[\left(\frac{\ln (\tau)-\eta}{\xi}\right)^{2}\right], \\
\xi & =\sqrt{\ln \left(1+\frac{\sigma^{2}}{\mu^{2}}\right)} \\
\eta & =\ln \left(\frac{\mu}{1+\left(\delta^{2} / \mu^{2}\right)}\right) .
\end{aligned}
$$

2.4. Improved Car-Following Model. According to the aforementioned idea, an improved car-following model that considers desired safety distance and the heterogeneity of driver's sensitivity is proposed and hereinafter referred to as the dynamic safety distance model (DSDM). Therefore, the DSDM is expressed as equations (5), (6), (11), and (12):

$$
a_{n}=\kappa(f(\tau))\left[V\left(\Delta x_{n}(t), h_{v}\right)-v_{n}\right]
$$

For equation (12), the following notes are provided:

(i) The improved model is governed by a new optimal velocity function, which is a function of the parameters of the maximum velocity, desired safety distance, and space headway. The maximum velocity is generally constant; thus, the optimal velocity depends on the desired safety distance and the space 
Table 1: Driver's sensitivity in different models.

\begin{tabular}{ccccc}
\hline Model & Bando et al. (OVM) & Helbing et al. (GFM) & Jiang et al. (FVDM) & Ge et al. (Ge's model) \\
\hline$\kappa$ & 1 & 0.85 & 0.41 & 2 \\
\hline
\end{tabular}

headway. Specifically, when the velocity increases, the desired safety distance increases synchronously. The desired safety distance varies with the variation in driving velocity. The improved model can better reveal the dynamic performance of car-following behavior than the conventional model.

(ii) The driver's sensitivity has heterogeneity. The sensitivity parameter of the improved model is not constant. The log-normal distribution is considered for analysis of the driver's sensitivity during carfollowing.

\section{Linear Stability Analysis}

Linear stability theory is applied to analyze the traffic flow model for obtaining the improved model's stability condition. We consider a system of $n$ vehicles on a unidirectional single-lane ring road of length $L$, and the traffic system is homogeneous. The vehicles are uniformly distributed with a constant space headway $h_{s}$ and velocity $v_{s}$ when the carfollowing system approaches the steady state. Then, the position of each vehicle in the steady-state traffic flow system at time $t$ is

$$
x_{n}^{0}(t)=h_{s} n+V\left(h_{s}\right) t .
$$

Assuming that the vehicle $n$ is disturbed, the position updates

$$
x_{n}(t)=x_{n}^{0}(t)+y_{n}(t)
$$

Equation (14) can be written as follows:

$$
y_{n}(t)=x_{n}(t)-x_{n}^{0}(t)
$$

The first and second derivatives of equation (15) are as follows:

$$
\begin{aligned}
& \dot{y}_{n}(t)=\dot{x}_{n}(t)-\dot{x}_{n}^{0}(t), \\
& \ddot{y}_{n}(t)=\ddot{x}_{n}(t) .
\end{aligned}
$$

The steady-state flow can accord with the following conditions:

$$
\begin{aligned}
\Delta \dot{x}_{n} & =0 ; \\
x_{n} & =0 ; \\
\Delta x_{n} & =h_{s} ; \\
\dot{x}_{n} & =v_{s} ; \\
V\left(\Delta x_{n}, h_{v}\right) & =V\left(h_{s}\right)=v_{s} .
\end{aligned}
$$

Then, the generic expression of the steady-state velocity is given by the following equation:

$$
v_{s}=\frac{v_{\max }}{2}\left[\tanh \left(h_{s}-h_{v}\right)-\tanh \left(h_{v}\right)\right] .
$$

Given that the steady-state value of $\Delta \dot{x}_{n}$ is 0 , an equivalent optimal velocity function is defined and given by equation (19) with $h_{v 0}=v_{s} t_{0}+h_{0}$ to facilitate the linear stability analysis. $h_{v 0}$ is the safety distance in steady state.

$$
v(\Delta x)=\frac{v_{\max }}{2}\left[\tanh \left(\Delta x-h_{v 0}\right)-\tanh \left(h_{v 0}\right)\right] .
$$

Each vehicle has an identical velocity, and the braking distances of the preceding and following vehicles are equal. According to equation (5), the desired safety distance is derived as follows:

$$
h_{v}=v\left(h_{s}\right) t_{0}+h_{0}+\dot{y}_{n} t_{0}=h_{v 0}+\dot{y}_{n} t_{0}
$$

In terms of the method of Xin et al. [26], by substituting equations (13), (14), and (20) into equation (6) and transforming the result with equation (19), then the optimal velocity can be derived as follows:

$$
\begin{aligned}
V\left(\Delta x_{n}, h_{v}\right) & =V\left(\Delta x_{n}^{0}+\Delta y_{n}\right)=V\left(h_{s}+\Delta y_{n}\right) \\
& =v\left(h_{s}+\Delta y_{n}-\dot{y}_{n} t_{0}\right)-v\left(-\dot{y}_{n} t_{0}\right) .
\end{aligned}
$$

By expanding $v\left(h_{s}+\Delta y_{n}-\dot{y}_{n} t_{0}\right)$ around $h_{s}$ and $v\left(-\dot{y}_{n} t_{0}\right)$ around 0 , the following equation can be obtained:

$$
V\left(h_{s}+\Delta y_{n}\right)=v\left(h_{s}\right)+v^{\prime}\left(h_{s}\right)\left(\Delta y_{n}-\dot{y}_{n} t_{0}\right)+v^{\prime}(0) \dot{y}_{n} t_{0},
$$

where $v^{\prime}\left(h_{s}\right)$ denotes the derivative of the optimal velocity at $h_{s}$ and the same is $v^{\prime}(0)$ which denotes the derivative of the optimal velocity at $h_{0}$.

By introducing equations (13)-(16), (21), and (22) into equation (12), the linearized equation (12) can be expressed as

$$
\ddot{y}_{n}=\kappa\left[v^{\prime}\left(h_{s}\right)\left(\Delta y_{n}-\dot{y}_{n} t_{0}\right)+v \prime(0) \dot{y}_{n} t_{0}-\dot{y}_{n}\right],
$$

with $\Delta y_{n}(t)=y_{n-1}(t)-y_{n}(t)$ and $\Delta \dot{y}_{n}(t)=\dot{y}_{n-1}-\dot{y}_{n}(t)$. Let $v^{\prime}\left(h_{s}\right)=r_{1} ; v^{\prime}(0) \approx 0$, and then, equation (23) can be written as follows:

$$
\ddot{y}_{n}=\kappa\left[r_{1} \Delta y_{n}-\left(r_{1} t_{0}+1\right) \dot{y}_{n}\right] .
$$

By expanding $y_{n}(t)=e^{j \alpha_{k} n+z t}$, the following equations are derived:

$$
\begin{aligned}
\dot{y}_{n}(t) & =z e^{j \alpha_{k} n+z t}, \\
\ddot{y}_{n}(t) & =z^{2} e^{j \alpha_{k} n+z t}, \\
\Delta y_{n}(t) & =z^{2} e^{j \alpha_{k} n+z t}\left(e^{\alpha_{\kappa} j}-1\right) .
\end{aligned}
$$

Substituting equation (25) into equation (24) yields

$$
z^{2}+z\left[\kappa\left(r_{1} t_{0}+1\right)\right]-\kappa r_{1}\left(e^{\alpha_{\kappa} j}-1\right)=0 .
$$

By substituting $z=\lambda+\mathrm{j} \omega$ into equation (26), the following equation is derived: 


$$
\lambda^{2}-\omega^{2}+2 \lambda \omega j+(\lambda+\omega j) \times \kappa \times\left(r_{1} t_{0}+1\right)-\kappa r_{1}\left(e^{\alpha_{\kappa} j}-1\right)=0 .
$$

Let $\lambda=0$ to obtain the following:

$$
-\omega^{2}-\kappa r_{1}\left(e^{\alpha_{\kappa} j}-1\right)+j \omega \kappa\left(r_{1} t_{0}+1\right)=0 .
$$

The real and imaginary parts of equation (28) are both 0 , which is the condition of the solution. It can be solved as follows:

$$
\kappa=r_{1} \frac{\left(1+\cos \alpha_{k}\right)}{\left(r_{1} t_{0}+1\right)^{2}} .
$$

Let $\alpha_{k} \longrightarrow 0$ to obtain the critical stability condition of the proposed car-following model:

$$
\kappa>\kappa_{s}=\frac{2 r_{1}}{\left(r_{1} t_{0}+1\right)^{2}} .
$$

This stable condition is consistent with that in the study by Peng et al. [43] when $t_{0}=0$. In equation (30), the vertex gradually decreases, and the stability domain gradually increases with the increase in $t_{0}$. The critical stability curves for the different model are shown in Figure 2. The diagram depicts that the stability region of the proposed model increases compared with that of the OVM. Furthermore, the instability decreases with the increment in $t_{0}$ because the desired safety distance increases synchronously. That is, larger $t_{0}$ corresponds to longer desired safety distance and more benefits of the following vehicle adjustment velocity.

\section{Numerical Simulation}

In this section, the numerical simulation experiments for the traffic flow evolution with a small initial disturbance are discussed under open boundary conditions. The simulation conditions in the study of Bando et al. [10] are adopted to facilitate comparative analysis. The parameters are determined by taking vehicle number $n=100$ and circuit length $L=200$ and $v_{\max }=2$. At the initial time, all vehicles are uniformly distributed with the identical space headway on the road. A small disturbance is exerted on the designated vehicle. The initial conditions are supposed as follows:

$$
\left\{\begin{array}{l}
\Delta x_{n}(0)=2, \text { for, } \quad n \neq 49,50, \\
\Delta x_{n}(0)=2-0.5, \text { for, } \quad n=49, \\
\Delta x_{n}(0)=2+0.5, \text { for, } \quad n=50 .
\end{array}\right.
$$

In the simulation, the position and velocity of each vehicle are updated using the following strategies:

$$
\begin{aligned}
& v_{n}(t+\Delta t)=v_{n}(t)+a_{n} \Delta t, \\
& x_{n}(t+\Delta t)=x_{n}(t)+v_{n}(t) \Delta t+\frac{1}{2} a_{n} \Delta t^{2},
\end{aligned}
$$

where the time step of the simulation is $\Delta t=0.1 \mathrm{~s}$.

4.1. Dynamic Performance of DSDM. In this section, the effect of the DSDM on spatial-time evolution of velocity and

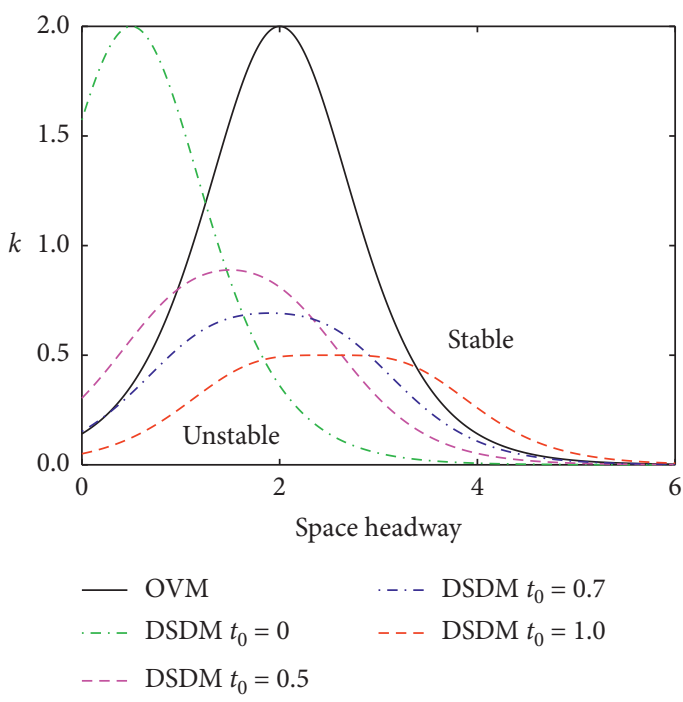

FIgure 2: Critical stability curves for the different models: the parameter of the OVM is $h_{c}=2$; the parameters of the DSDM are $h_{v}$ when $t_{0}=0,0.5,0.7,1.0$.

space headway is invested by numerical simulation. Equations (4) and (5) show that more extensive $t_{0}$ indicates a larger desired safety distance. According to the stability condition equation (30), more extensive $t_{0}$ means a larger stability region. However, a considerable response time is not beneficial to traffic efficiency. Therefore, we attempt to discuss the performance of the DSDM for different values of $t_{0}=0,0.5,0.7$, and 1.0 when $\kappa$ obeys a log-normal distribution.

Figure 3 indicates that the temporal and spatial evolutions of velocities gradually become stable, and the oscillation range is reduced as $t_{0}$ equals $0.5,0.7$, and 1.0. Figure $3(\mathrm{~d})$ plots that the amplitude of the velocity waves dissipates after time $t=1500$ when $t_{0}$ equals 1.0. Therefore, increasing the value of $t_{0}$ could improve the stability of the traffic flow and alleviate traffic congestion. In other words, adequate desired safety distance can improve the stability of the traffic flow.

However, the steady velocity tends to decrease as the value of $t_{0}$ increases. For instance, Figure 3 shows that the stable velocities are approximately $1.9,1.6,1.5$, and 1.3 when $t_{0}$ values are $0,0.5,0.7$, and 1.0, respectively. Therefore, a longer response time implies lower traffic efficiency. Driving experience also shows that the longer the driver's reaction time is, the less favorable it is to keep driving at high velocity. Therefore, the value of the response time $t_{0}$ should be as large as possible within relatively small bounds to enhance traffic stability.

Figure 4 shows consistency with Figure 3. The fluctuation of the space headway is also reduced with the increase in $t_{0}$. When $t_{0}=1.0$, the curve of the space headway becomes stable. The traffic flow with larger $t_{0}$ is more stable.

The abovementioned analyses reveal that the amplitude of velocity and space headway profiles gradually stabilizes with the increase in $t_{0}$. The parameter $t_{0}$ equal to 1.0 is approximate to the $99 \%$ quantile of the actual expected response time 0.81 [42]. Therefore, DSDM adopts $t_{0}=1$ to 


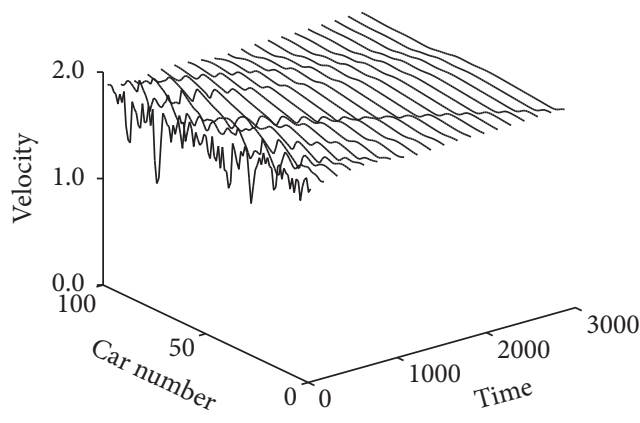

(a)

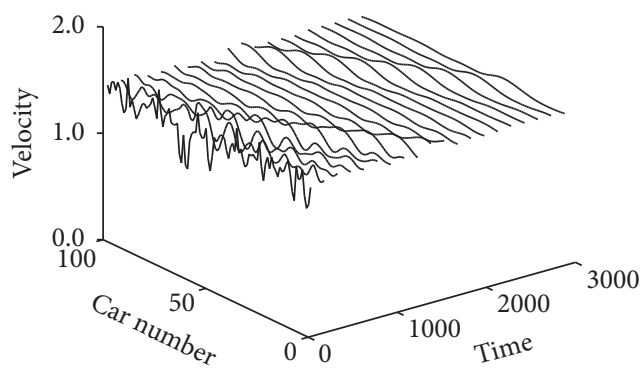

(c)

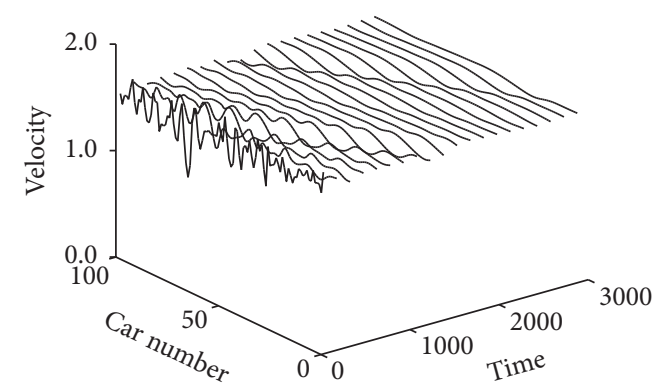

(b)

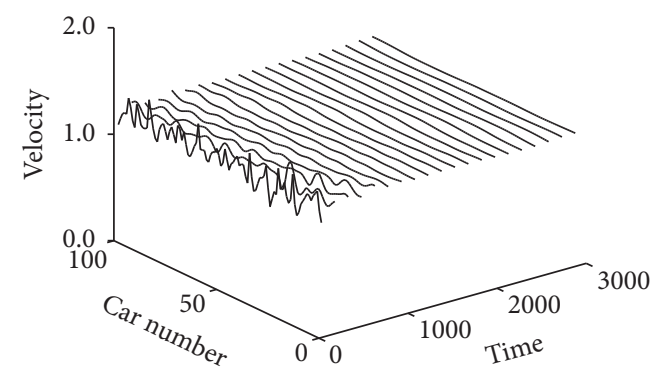

(d)

FIGURE 3: Temporal and spatial velocities when $t_{0}=0,0.5,0.7$, and 1.0. (a) $t_{0}=0$. (b) $t_{0}=0.5$. (c) $t_{0}=0.7$. (d) $t_{0}=1.0$.

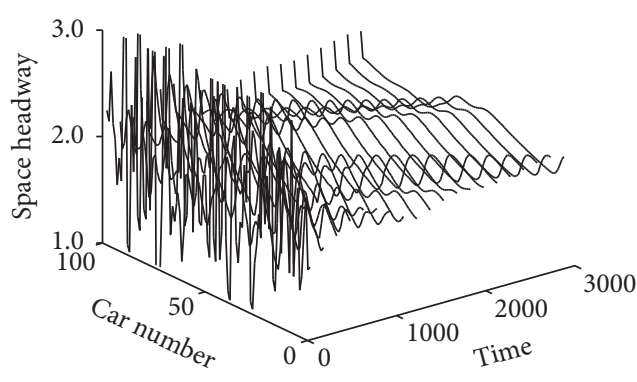

(a)

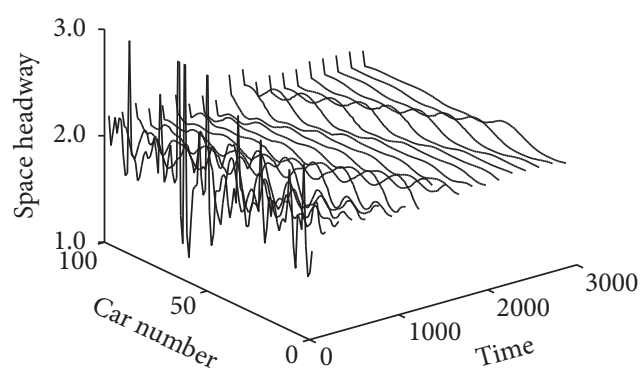

(c)

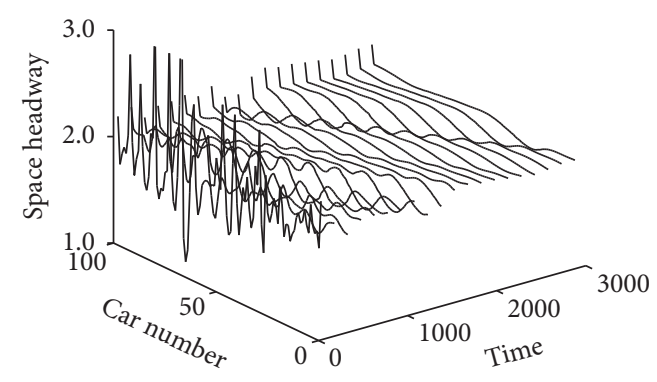

(b)

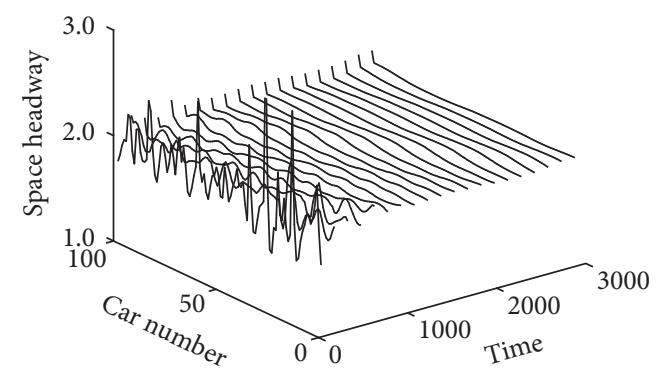

(d)

Figure 4: Temporal and spatial space headway when $t_{0}=0,0.5,0.7$, and 1.0. (a) $t_{0}=0$. (b) $t_{0}=0.5$. (c) $t_{0}=0.7$. (d) $t_{0}=1.0$.

ensure traffic stability and efficiency. In conclusion, DSDM with proper parameter contributes to the improvement in the traffic flow stability and the avoidance of the unstable traffic phenomenon.

\subsection{Comparative Analysis of DSDM and OVM}

4.2.1. Influence of the Desired Safety Distance. In this section, the influence of the desired safety distance on the evolution of velocity and space headway is first discussed. The simulation conditions are the same as those in the study of Bando et al. [10], and the parameters are listed in Table 2. Notably, the parameter $\kappa=1$ is set in different models in order to analyze the influence of the desired safety distance.

Figures 5 and 6 illustrate the velocity and the space headway profiles of different car-following models at time step $t=100,500,1500$, and 3000. Figure 5(a) indicates that the vehicles stop and go and the velocities fluctuate in the 
TABLE 2: Parameters of the numerical simulation experiment when $\kappa$ is constant.

\begin{tabular}{llcc}
\hline Model & $\kappa$ & $t_{0}$ & Safety distance \\
\hline OVM & 1 & - & 2 \\
DSDM & 1 & 1 & $h_{v}$ \\
\hline
\end{tabular}

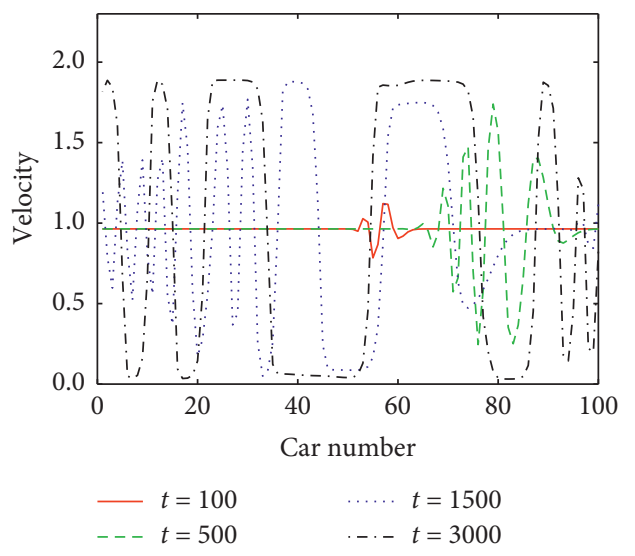

(a)

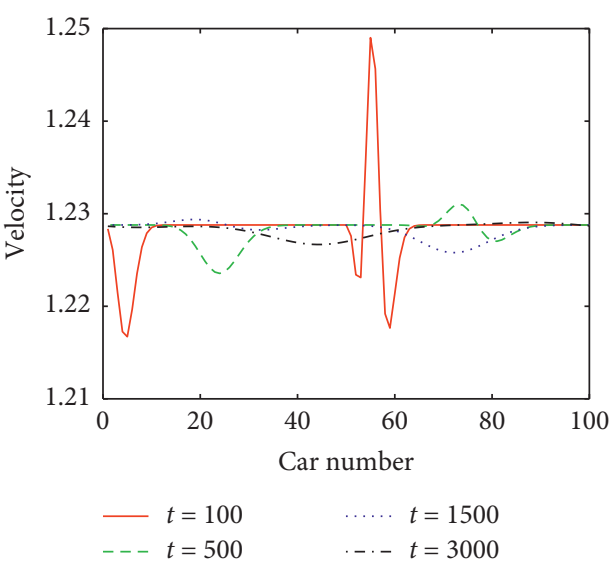

(b)

Figure 5: Profiles of velocities at time step $t=100,500,1500$, and 3000 when $\kappa$ is a constant. (a) OVM. (b) DSDM.

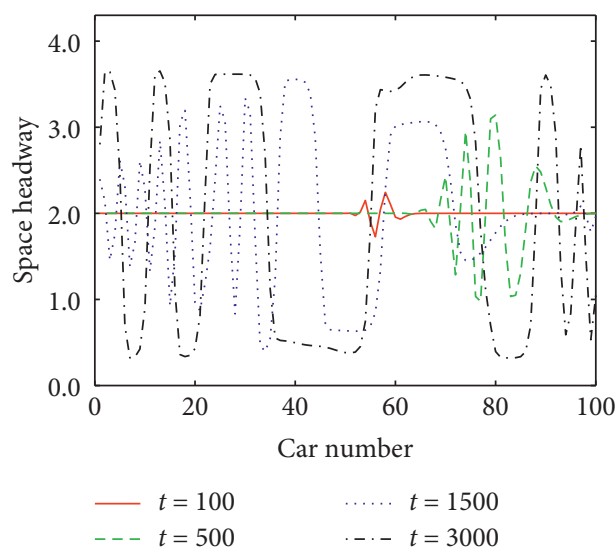

(a)

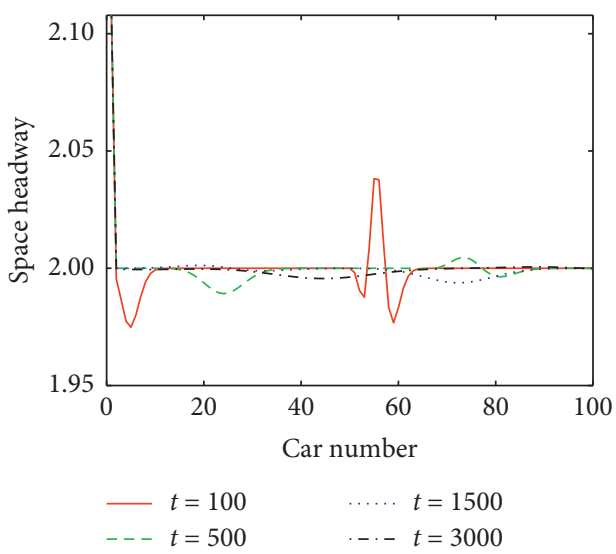

(b)

Figure 6: Profiles of space headway at time step $t=100,500,1500$, and 3000 when $\kappa$ is a constant. (a) OVM. (b) DSDM.

range of $[0,2]$ after disturbance in the OVM. Figure 5(b) shows that the propagation of disturbance gradually converges over time in the DSDM. The velocities fluctuate within a small range from 1.21 to 1.25 and finally stabilize around the average velocity of 1.23 .

The profile of Figure 6 is consistent with that of Figure 5. Figure 6(a) indicates that the oscillation amplitude of space headway gradually increases in the OVM, and it ranges from 0 to 4 . However, Figure 6(b) suggests that the oscillation amplitude of the space headway profile is relatively small considering the desired safety distance. The space headway fluctuates from 1.95 to 2.10 and can quickly stabilize at the approximate average value of 2 .

The profiles of Figures 5 and 6 show that considering the desired safety distance can effectively suppress traffic congestion and improve traffic flow stability. The reason is that the optimal velocity output is based on the function of the space headway and the safety distance. The safety distance in the OVM is a preset constant and deviates from the actual value as the velocity changes. However, the desired safe distance in the DSDM is updated according to velocity, and this condition can result in a smaller deviation from the real-time space headway than that of the OVM. Therefore, the DSDM can output a more smooth optimal velocity to improve the stability of the traffic flow.

4.2.2. Influence of the Driver's Sensitivity. In this section, the DSDM and the OVM are compared to analyze the effect of the driver's sensitivity on velocity and space headway 
TABle 3: Parameters of numerical simulation experiment when $\kappa$ obeys log-normal distribution.

\begin{tabular}{lccc}
\hline Model & $\kappa$ & $t_{0}$ & Safety distance \\
\hline OVM & $\kappa(f(\tau))$ & - & 2 \\
DSDM & $\kappa(f(\tau))$ & 1 & $h_{v}$ \\
\hline
\end{tabular}

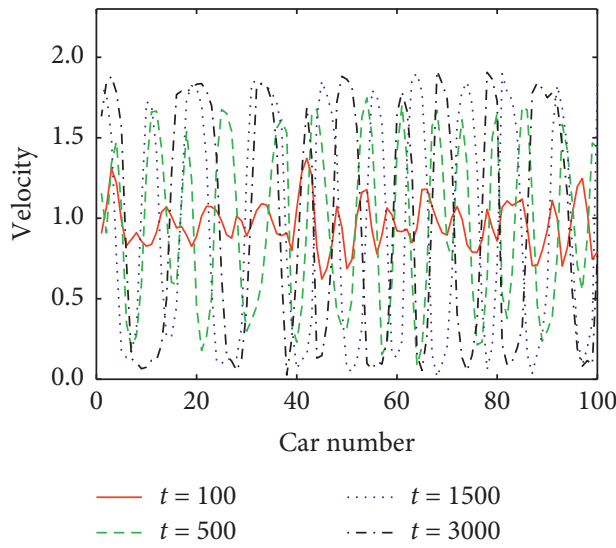

(a)

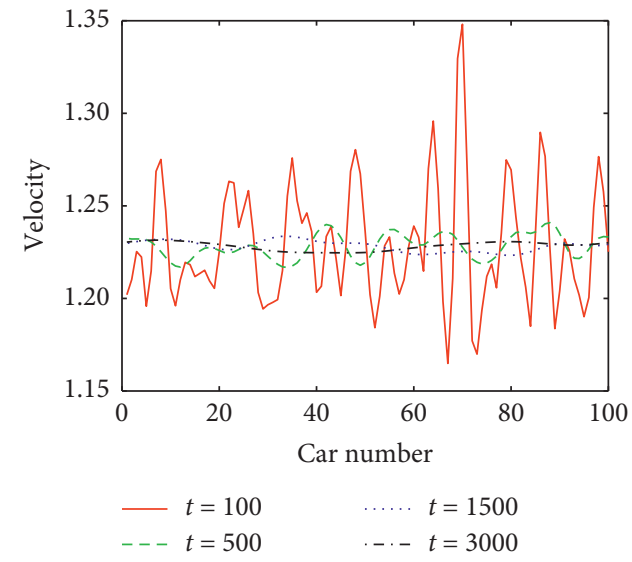

(b)

Figure 7: Profiles of velocities at time step $t=100,500,1500$, and 3000 when $\kappa$ obeys log-normal distribution. (a) OVM. (b) DSDM.

evolution on the basis of Section 4.2.1. The simulation conditions are the same as those in the study of Bando et al. [10]. The driver's sensitivity $\kappa$ obeys the log-normal distribution. The parameters are listed in Table 3 .

Figure 7(a) displays the velocity profiles when the driver's sensitivity in the OVM obeys the log-normal distribution. Compared with Figure 7(b), the velocity oscillation frequency in Figure 7(a) is strengthened, the oscillation range is $[0,2]$, the vehicles stop and go, and apparent traffic congestion is observed. This result can be explained that the extent to which drivers react to their respective leaders is actually changed by allowing sensitivity parameters to differ between drivers. Figure 7(b) shows that the velocity of vehicles changes smoothly after being disturbed. The velocity fluctuates in the interval of $[1.15,1.35]$ and finally stabilizes in a small range around 1.23 . The average velocity of the DSDM is $28 \%$ higher than that of the OVM. This finding can be explained as follows: the DSDM can keep a real-time desired safe distance and prevent the propagation of disturbance, which results in reducing the total frequency of vehicle stop and go. This condition is consistent with that of the jam-absorption driving strategy of $\mathrm{He}$ et al. [44]. Meanwhile, the intensity of deceleration has decreased. Furthermore, less possibility of being captured by traffic jam has the potential of improving traffic efficiency.

Moreover, the heterogeneity of driver's sensitivity will aggravate the disturbance of OVM, but it can be suppressed in the DSDM. This result is reasonable given that not only the extent to which drivers react to their respective leaders is made driver-dependent but also the desired safety distance drivers need to react. Real-time desired safety distance can be less of a deviation from the space headway than constant safety distance, which neutralizes the oscillation caused by the heterogeneity of the driver's sensitivity. In conclusion, the heterogeneity of driver's sensitivity can be compatible in the DSDM but not in OVM.

Figure 8(a) displays the space headway profiles when the driver's sensitivity in the OVM obeys the log-normal distribution. The oscillation interval of the space headway is $[-2,6]$, the oscillation amplitude is large, and the space headway is less than zero at some points. The phenomenon has been caused by the heterogeneity of driver's sensitivity, which indicates that the OVM cannot adapt to the heterogeneity. However, the amplitude of space headway fluctuation decreases to the interval of $[1.8,2.3]$, as shown in Figure 8(b), and the curves become more stable after time step 1500. This finding illustrates that the DSDM has good compatibility with the heterogeneity of driver's sensitivity because the DSDM allows the driver to maintain a more reasonably safe distance in real time to avoid collisions, but the OVM cannot. The inability of the driver to precisely predict the safety distance is addressed. Moreover, less deviation from the space headway suppresses the disturbance caused by the heterogeneity of the driver's sensitivity. In summary, the DSDM can adapt to the heterogeneity of driver's sensitivity and suppress traffic congestion.

Similarly, Figures 7(b), 5(a), 8(b), and 6(a) are compared. The results show that the oscillation intervals of the velocity and space headway of the DSDM at different time steps are smaller than those of the conventional OVM. The steadystate velocity of Figure 7(b) is higher than that of Figure 5(a). As observed, the performance of the DSDM is better than 


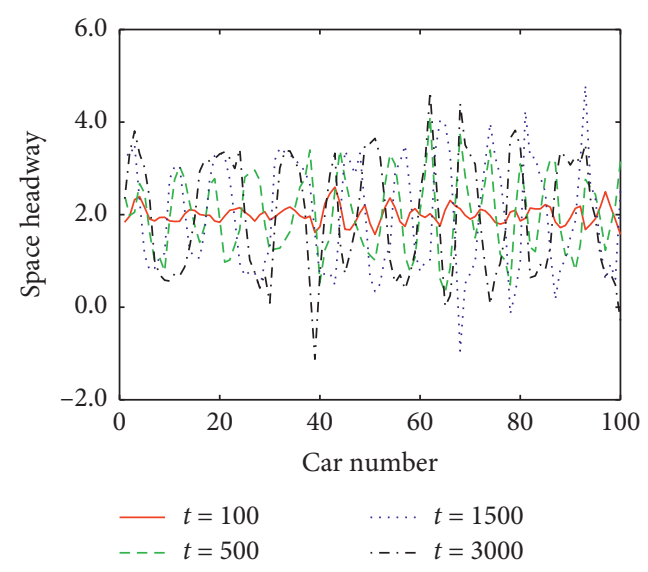

(a)

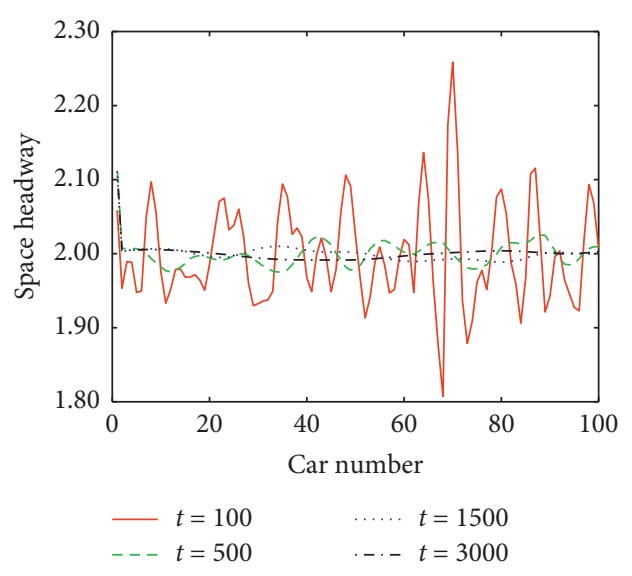

(b)

Figure 8: Profiles of space headway at time step $t=100,500,1500$, and 3000 when $\kappa$ obeys log-normal distribution. (a) OVM. (b) DSDM.

that of OVM. DSDM can improve traffic stability, alleviate traffic congestion, and be more compatible with the heterogeneity of driver's sensitivity than the OVM.

\section{Conclusions}

The driver needs to continually adjust the following distance to the safety distance according to the driver's driving experience during car-following. To quantify the abovementioned behavior dynamically, we modified the constant safety distance in the optimal velocity function to a dynamic desired safety distance. Then, we established a new optimal velocity function. The boundary conditions of the new optimal velocity function were analyzed theoretically. The driver's sensitivity was then set to obey the log-normal distribution to describe the driver's heterogeneity. Then, the improved car-following model considering the desired safety distance and the heterogeneity of driver's sensitivity was proposed.

Theoretic analytical and numerical simulation methods were used to explore the performance of the improved model. The linear stability criterion of the improved model was investigated. Numerical simulation was performed to study the properties of the DSDM. These results are summarized as follows:

(i) The DSDM can suppress the propagation and amplification of traffic congestion and improve traffic flow stability.

(ii) The temporal and spatial velocities and space headway profiles of the DSDM are smoother than those of the OVM. Less traffic stop-and-go phenomenon could be found. The average velocity of traffic flow in DSDM is enhanced synchronously.

(iii) Meanwhile, the desired safety distance and the heterogeneity of driver's sensitivity are allowed in DSDM. This case is consistent with the realistic scenarios where the driver of the following vehicle continuously adjusts the safety distance to the preceding vehicle by some criteria. The maintenance of the desired safety distance neutralizes the disturbance caused by the heterogeneity of driver's sensitivity. Overall, theoretical analysis and numerical simulation show that the DSDM can still improve traffic stability while maintaining compatibility with the heterogeneity of driver's sensitivity.

This research contributes to modeling realistic longitudinal driving behaviors with traffic stability and individual heterogeneity. However, quantifying the heterogeneity of driver's sensitivity with response time distribution cannot be very satisfactory. The driver's sensitivity is also related to other factors, such as space headway and speed. More precise quantification of this parameter is essential. In addition, we investigate the dynamic performance of the improved model through numerical simulation. More field observation data should be used to test the reliability of the improved model. Particularly, calibrating and validating car-following models containing the heterogeneity of driver's sensitivity will be challenging [45]. This research area would contribute substantially to better understanding of the dynamic performance of car-following systems.

\section{Data Availability}

The simulation result data used to support the findings of this study are available from the corresponding author upon reasonable request.

\section{Conflicts of Interest}

The authors declare that they have no conflicts of interest.

\section{Acknowledgments}

This research was supported by the National Natural Science Foundation of China, Grant no. 71871029, the Fundamental Research Funds for the Central Universities, CHD, Grant nos. 300102210301 and 300102219306, Natural Science Basic Research Program of Shaanxi, Grant no. 2020JM-222, and 
the 111 Project of Sustainable Transportation for Urban Agglomeration in Western China (no. B20035).

\section{References}

[1] D. Helbing, "Derivation of non-local macroscopic traffic equations and consistent traffic pressures from microscopic car-following models," The European Physical Journal B, vol. 69, no. 4, pp. 539-548, 2009.

[2] Y. S. Qian, X. M. Shao, J. W. Zeng, and M. Wang, "An improved cellular automaton model with the consideration of a multi-point tollbooth," Physica A: Statistical Mechanics and Its Applications, vol. 392, no. 23, pp. 5874-5878, 2013.

[3] D. Ngoduy, "Application of gas-kinetic theory to modelling mixed traffic of manual and ACC vehicles," Transportmetrica, vol. 8, no. 1, pp. 43-60, 2012.

[4] P. Redhu and A. K. Gupta, "Delayed-feedback control in a Lattice hydrodynamic model," Communications in Nonlinear Science and Numerical Simulation, vol. 27, no. 1-3, pp. 263270, 2015.

[5] A. Reuschel, "Vehicle movements in a platoon," Oesterreichisches Ingenieeur-Archir, vol. 4, pp. 193-215, 1950.

[6] L. A. Pipes, "An operational analysis of traffic dynamics," Journal of Applied Physics, vol. 24, no. 3, pp. 274-281, 1953.

[7] E. Kometani and T. Sasaki, "On the Stability of traffic flow," Journal of Operations Research Japan, vol. 2, no. 1, pp. 11-26, 1958.

[8] C. Kikuchi and P. Chakroborty, "Fuzzy neural network control of complex systems: a study on longitudinal vehicle control," Procedings of the World Congress on Neural Networks, vol. 3, pp. 84-92, 1992.

[9] G. F. Newell, "Nonlinear effects in the dynamics of car following," Operations Research, vol. 9, no. 2, pp. 209-229, 1961.

[10] M. Bando, K. Hasebe, A. Nakayama, A. Shibata, and Y. Sugiyama, "Dynamical model of traffic congestion and numerical simulation," Physical Review E, vol. 51, no. 2, pp. 1035-1042, 1995.

[11] M. Treiber, A. Hennecke, and D. Helbing, "Congested traffic states in empirical observations and microscopic simulations,” Physical Review E, vol. 62, no. 2, pp. 1805-1824, 2000.

[12] Z. He, L. Zheng, and W. Guan, "A simple nonparametric carfollowing model driven by field data," Transportation Research Part B: Methodological, vol. 80, pp. 185-201, 2015.

[13] D. Helbing and B. Tilch, "Generalized force model of traffic dynamics," Physical Review E, vol. 58, no. 1, pp. 133-138, 1998.

[14] R. Jiang, Q. Wu, and Z. Zhu, "Full velocity difference model for a car-following theory," Physical Review E, vol. 64, no. 1, pp. 133-138, 2001.

[15] S. Jiao, S. Zhang, B. Zhou et al., "Dynamic performance and safety analysis of car-following models considering collision sensitivity," Physica A: Statistical Mechanics and Its Applications, vol. 564, Article ID 125504, 2021.

[16] S. Jiao, S. Zhang, Z. Li, B. Zhou, and D. Zhao, “An improved car-following speed model considering speed of the lead vehicle, vehicle spacing, and driver's sensitivity to them," Journal of Advanced Transportation, vol. 2020, no. 2, 13 pages, Article ID 2797420, 2020.

[17] Y. Peng, S. Liu, and D. Z. Yu, "An improved car-following model with consideration of multiple preceding and following vehicles in a driver's view," Physica A: Statistical Mechanics and Its Applications, vol. 538, Article ID 122967, 2020.

[18] S. Yu, X. Zhao, Z. Xu, and Z. Shi, “An improved car-following model considering the immediately ahead car's velocity difference," Physica A: Statistical Mechanics and Its Applications, vol. 461, pp. 446-455, 2016.

[19] X. Zhao and Z. Gao, "A new car-following model: full velocity and acceleration difference model," The European Physical Journal B, vol. 47, no. 1, pp. 145-150, 2005.

[20] H. Song, H. Ge, F. Chen, and R. Cheng, "TDGL and mKdV equations for car-following model considering traffic jerk and velocity difference," Nonlinear Dynamics, vol. 87, no. 3, pp. 1809-1817, 2016.

[21] T. Nagatani and R. Takashi, "Stabilization and enhancement of traffic flow by the next-nearest-neighbor interaction," Physical Review E, vol. 60, no. 6, pp. 6395-6401, 1999.

[22] H. Lenz, C. K. Wagner, and R. Sollacher, "Multi-anticipative car-following model," The European Physical Journal B, vol. 7, no. 2, pp. 331-335, 1999.

[23] H. X. Ge, S. Q. Dai, L. Y. Dong et al., “'“Stabilization effect of traffic flow in an extended car-following model based on an intelligent transportation system application," (in English)," Physical Review E, vol. 70, no. 6, Article ID 066134, 2004.

[24] A. Nakayama, Y. Sugiyama, and K. Hasebe, "Effect of looking at the car that follows in an optimal velocity model of traffic flow," Physical Review E Statistical Nonlinear \& Soft Matter Physics, vol. 65, no. 1, Article ID 016112, 2002.

[25] K. Hasebe, A. Nakayama, and Y. Sugiyama, "Dynamical model of a cooperative driving system for freeway traffic," Physical Review E Statistical Nonlinear \& Soft Matter Physics, vol. 68, no. 2, Article ID 026102, 2003.

[26] Q. Xin, N. Yang, R. Fu, S. Yu, and Z. Shi, "Impacts analysis of car following models considering variable vehicular gap policies," Physica A: Statistical Mechanics and Its Applications, vol. 501, pp. 338-355, 2018.

[27] R. Cheng, H. Ge, and J. Wang, "An extended continuum model accounting for the driver's timid and aggressive attributions," Physics Letters A, vol. 381, no. 15, pp. 1302-1312, 2017.

[28] D. W. Liu, Z. K. Shi, and W. H. Ai, “"'An improved carfollowing model from the perspective of driver's forecast behavior," International Journal of Modern Physics C, vol. 28, no. 4, Article ID 1750046, 2017.

[29] Q. Zhai, H. Ge, and R. Cheng, "An extended continuum model considering optimal velocity change with memory and numerical tests," Physica A: Statal Mechanics and Its Applications, vol. 490, pp. 774-785, 2017.

[30] C. Zhai and W. T. Wu, "An extended continuum model with consideration of the self-anticipative effect," Modern Physics Letters B, vol. 32, no. 31, Article ID 1850382, 2018.

[31] R. Cheng, H. Ge, and J. Wang, "An extended macro traffic flow model accounting for multiple optimal velocity functions with different probabilities," Physics Letters A, vol. 381, no. 32, pp. 2608-2620, 2017.

[32] L. Yu, Z.-k. Shi, and T. Li, "A new car-following model with two delays," Physics Letters A, vol. 378, no. 4, pp. 348-357, 2014.

[33] S. Ossen and S. P. Hoogendoorn, "“Driver heterogeneity in car following and its impact on modeling traffic dynamics," transportation research record," Journal of the Transportation Research Board, vol. 1999, no. 1, pp. 95-103, 2007.

[34] Z. Li, X. Xu, S. Xu, and Y. Qian, "A heterogeneous traffic flow model consisting of two types of vehicles with different sensitivities," Communications in Nonlinear Science and Numerical Simulation, vol. 42, pp. 132-145, 2017.

[35] S. Ossen and S. P. Hoogendoorn, "Heterogeneity in car-following behavior: theory and empirics," Transportation 
Research Part C: Emerging Technologies, vol. 19, no. 2, pp. 182-195, 2011.

[36] G. Schultz and L. Rilett, "Analysis of distribution and calibration of car-following sensitivity parameters in microscopic traffic simulation models," Transportation Research Record Journal of the Transportation Research Board, vol. 1876, pp. 41-51, 2004.

[37] M. Makridis, L. Leclercq, B. Ciuffo et al., "Formalizing the heterogeneity of the vehicle-driver system to reproduce traffic oscillations," Transportation Research Part C: Emerging Technologies, vol. 120, Article ID 102803, 2020.

[38] D. L. Gerlough and M. J. Huber, Traffic-flow Theory: A Monograph, Transportation Research Board, National Research Council, Washington, DC, USA, 1975.

[39] M. Bando, K. Hasebe, K. Nakanishi, and A. Nakayama, "Analysis of optimal velocity model with explicit delay," Physical Review E, vol. 58, no. 5, pp. 5429-5435, 1998.

[40] H. X. Ge, X. P. Meng, J. Ma et al., "An improved car-following model considering influence of other factors on traffic jam," Physics Letters A, vol. 377, no. 1-2, pp. 9-12, 2012.

[41] J. Zheng, K. Suzuki, and M. Fujita, "Car-following behavior with instantaneous driver-vehicle reaction delay: a neuralnetwork-based methodology," Transportation Research Part C: Emerging Technologies, vol. 36, pp. 339-351, 2013.

[42] N. Gartner, C. Messer, and A. Rathi, Traffic Flow Theory: A State-Of-The-Art Report: Revised Monograph on Traffic Flow Theory, US. Department of transportation, Washington, DC, USA, 2002.

[43] G. H. Peng, X. H. Cai, C. Q. Liu, B. F. Cao, and M. X. Tuo, "Optimal velocity difference model for a car-following theory," Physics Letters A, vol. 375, no. 45, pp. 3973-3977, 2011.

[44] Z. He, L. Zheng, L. Song, and N. Zhu, "A jam-absorption driving strategy for mitigating traffic oscillations," IEEE Transactions on Intelligent Transportation Systems, vol. 18, no. 4, pp. 802-813, 2017.

[45] M. Saifuzzaman and Z. Zheng, "Incorporating human-factors in car-following models: a review of recent developments and research needs," Transportation Research Part C: Emerging Technologies, vol. 48, pp. 379-403, 2014. 